Research report

\title{
Task difficulty modulates brain activation in the emotional oddball task
}

\author{
Rachel E. Siciliano ${ }^{a}$, David J. Madden ${ }^{a, b, *}$, Catherine W. Tallman ${ }^{a}$, Maria A. Boylan ${ }^{a}$, Imke Kirste ${ }^{a}$, \\ Zachary A. Monge $^{c}$, Lauren E. Packard ${ }^{a}$, Guy G. Potter ${ }^{\mathrm{a}, \mathrm{b}}$, Lihong Wang ${ }^{\mathrm{d}}$
}

a Brain Imaging and Analysis Center, Duke University Medical Center, Durham, NC 27710, United States

${ }^{\mathrm{b}}$ Department of Psychiatry and Behavioral Sciences, Duke University Medical Center, Durham, NC 27710, United States

${ }^{\mathrm{c}}$ Center for Cognitive Neuroscience, Duke University, Durham, NC 27708, United States

${ }^{\mathrm{d}}$ Department of Psychiatry, University of Connecticut Health Center, Farmington, CT 06030, United States

\section{A R T I C L E I N F O}

\section{Article history:}

Received 6 November 2016

Received in revised form 9 March 2017

Accepted 27 March 2017

Available online 1 April 2017

\section{Keywords:}

Attention

Distraction

Emotion

Task difficulty

Cognitive control

fMRI

\begin{abstract}
A B S T R A C T
Previous functional magnetic resonance imaging (fMRI) studies have reported that task-irrelevant, emotionally salient events can disrupt target discrimination, particularly when attentional demands are low, while others demonstrate alterations in the distracting effects of emotion in behavior and neural activation in the context of attention-demanding tasks. We used fMRI, in conjunction with an emotional oddball task, at different levels of target discrimination difficulty, to investigate the effects of emotional distractors on the detection of subsequent targets. In addition, we distinguished different behavioral components of target detection representing decisional, nondecisional, and response criterion processes. Results indicated that increasing target discrimination difficulty led to increased time required for both the decisional and nondecisional components of the detection response, as well as to increased targetrelated neural activation in frontoparietal regions. The emotional distractors were associated with activation in ventral occipital and frontal regions and dorsal frontal regions, but this activation was attenuated with increased difficulty. Emotional distraction did not alter the behavioral measures of target detection, but did lead to increased target-related frontoparietal activation for targets following emotional images as compared to those following neutral images. This latter effect varied with target discrimination difficulty, with an increased influence of the emotional distractors on subsequent target-related frontoparietal activation in the more difficult discrimination condition. This influence of emotional distraction was in addition associated specifically with the decisional component of target detection. These findings indicate that emotion-cognition interactions, in the emotional oddball task, vary depending on the difficulty of the target discrimination and the associated limitations on processing resources.
\end{abstract}

(c) 2017 Elsevier B.V. All rights reserved.

\section{Introduction}

Emotional stimuli affect how we process and respond to our surroundings. However, as Phelps (2006) has noted, despite this generally accepted tenet, human cognition research often regards emotion and cognition as separate realms. Yet, both behavioral evidence and functional magnetic resonance imaging (fMRI) results demonstrate that the two are inextricably intertwined. Emotional stimuli typically activate subcortical regions such as the amygdala, ventral striatum, and thalamus, but also a network of cortical regions including lateral and medial prefrontal cortex and the anterior cingulate, as well as sensory regions (Dolcos et al., 2011a,b; Kober et al., 2008; Mather and Carstensen, 2003).

\footnotetext{
* Corresponding author at: Brain Imaging and Analysis Center, Box 3918, Duke University Medical Center, Durham, NC 27710, United States.

E-mail address: david.madden@duke.edu (D.J. Madden).
}

Emotional stimuli, particularly those expressing anger, fear, or disgust, can be highly salient and thus appear to be processed without requiring much attentional capacity, consistent with a predominantly subcortical pattern of activation (Anderson et al., 2003; Morris et al., 1999). However, fMRI studies that have manipulated the attentional demands of the task, in conjunction with the emotional valence of the stimuli, have led to a more complex view in which processing of the emotional stimuli is constrained by the attentional demands of the task. Specifically, several studies, both event-related potential (ERP) and fMRI, have found that the distracting effects of emotional stimuli, even those expressing fear, a negative emotion with high valence, are substantially reduced in the context of an attention-demanding task (Doallo et al., 2006; Lange et al., 2003; Pessoa et al., 2002a,b), while others demonstrate that attention to threatening stimuli is prioritized (Hartikainen et al., 2012; Schmidt et al., 2015). There is also evidence that emotional distraction is processed both automatically 
and modulated by attentional demands, yet emotion and attentional load interacted only when emotional charge and processing demands were more finely assessed. For example, distracting effects of emotion were largest with low perceptual load, long presentation duration, and images with high emotional (as compared to low emotional) content (Shafer et al., 2012). This array of results highlights the importance of manipulating both emotional valence and attentional load in order to clarify differences in emotionattention interactions as a function of task difficulty.

The emotional oddball task (Wang et al., 2005, 2006; Wang et al., 2008a,b,c) yields information regarding the interaction of emotion and attention by measuring a detection response to relatively rare (oddball) targets, in the context of more frequently occurring standards and rarely occurring distractors. When the distractors are images with an emotional valence, they can lead to a distinct pattern of neural activation, even though neither the standards nor the distractors require an overt response, and allow for the estimation of the cognitive processes engaged in response to task-irrelevant emotional stimuli. Wang et al. (2005), for example, reported that distractors that were sad images (e.g., related to grief and despair) activated ventral brain regions, including the amygdala, fusiform gyrus, and inferior frontal gyrus, relative to neutral images (phase scrambled pictures), whereas the detection of targets (circles) activated dorsal regions of frontal, parietal, and cingulate cortex.

In the present experiment we sought to determine whether the brain activation associated with sad images, in the emotional oddball task (Wang et al., 2005), varied as a function of attentional load (Doallo et al., 2006; Shafer et al., 2012), in terms of target discrimination difficulty, in order to determine if activation to emotional images is constrained by task demands, therefore presumably under the control of top-down regulation, or demonstrates no difference between target discrimination difficulty conditions, suggesting that sad emotional stimuli are given priority regardless of attentional demands. We also seek to extend previous research demonstrating the effect of target discrimination difficulty on high valence emotional stimuli, for example, fear, by determining if these findings hold true for low valence emotional stimuli, specifically sadness. In addition, the emotional oddball task is sensitive to individual differences in emotion regulation ability, as it is thought to require cognitive control in order to maintain performance in response to attentional targets despite the presence of emotional distractors, and the pattern of activation reflecting emotion-cognition interactions has been reported to vary for individuals with major depressive disorder (Wang et al., 2008c), mild traumatic brain injury, orbitofrontal cortex lesion patients, and patients with anxiety disorders (Bishop et al., 2004; Bishop, 2008; Maki-Marttunen et al., 2015, 2017). Thus, in this experiment we examined the relation between target-related activation and different aspects of target detection performance.

Many previous studies on distraction from emotional stimuli comprise tasks in which the task-relevant and emotional items are presented concurrently (Doallo et al., 2006; Lange et al., 2003; Pessoa et al., 2002a,b; Shafer et al., 2012) (but cf. Dolcos et al., 2008). The first goal of this experiment was to examine the influence of target discrimination difficulty on distraction from emotional stimuli. Specifically, for fMRI data, we examined the variation in task-irrelevant distractors of varying emotional valence (sad and neutral images), as a function of target discrimination difficulty. Also, within this goal, we examined the effects of emotional distractors on responses to subsequently occurring taskrelevant items (targets), as a function of target discrimination difficulty, in activation and behavioral performance. A useful feature of the emotional oddball task is that distractors and targets are separated in time, which allows estimation of the influence of the distractors on the detection of subsequently appearing targets.
Studies utilizing fMRI, electrophysiological, and behavioral measures where emotional stimuli preceded target presentation, have shown that task-irrelevant unpleasant emotional images interfere with subsequent target discrimination (Hartikainen et al., 2000, 2007). This paradigm extends previous findings to determine whether emotion-cognition interactions vary with target discrimination difficulty when emotional distraction may have an extended effect on subsequent cognitive processing, as well as whether target discrimination difficulty modulates the processing of emotional distractors, independently of the target.

A second goal of this experiment was to investigate the effects of target discrimination difficulty and emotional distraction on different components of target detection performance. We analyzed the behavioral data with a diffusion model of reaction time (RT) that distinguished the decisional and nondecisional components of the target detection response (Ratcliff et al., 2016; Voss et al., 2013; Wagenmakers et al., 2007). Previous investigations of emotional distraction effects, as in the majority of behavioral research, have measured task performance in terms of mean RT and accuracy (e.g., Doallo et al., 2006; Shafer et al., 2012). The diffusion model of RT uses estimates of the underlying RT distribution to distinguish several of the information processing components contributing to mean RT and accuracy, including the rate of information accumulation towards a decision (drift rate), visual encoding and motor response time (nondecision time), and response cautiousness (boundary separation). Whereas these RT model parameters have been found to correlate with structural properties of the brain (white matter integrity; Madden et al., 2009; Yang et al., 2015), to our knowledge the relation between task-related brain activation and RT diffusion model parameters has not been reported previously.

Previous studies have found that increasing attentional load decreases the influence of emotional stimuli (Doallo et al., 2006; Kurth et al., 2016; Lange et al., 2003; Pessoa et al., 2002a,b), presumably because increasing attentional load decreases the processing resources available for task-irrelevant items. We thus hypothesized that, for healthy adults, increasing the difficulty of the target discrimination would decrease the neural activation associated with sad emotional distractors in the present emotional oddball task. To maximize the opportunity to detect an effect of target discrimination difficulty, in this experiment, we used a sample of older adult participants, under the assumption that, in view of age-related decline in fluid cognition and some (though not all) attentional abilities (Kramer and Madden, 2008; McAvinue et al., 2012; Monge and Madden, 2016), the effects of perceptual difficulty would be more clearly evident. Maylor and Lavie (1998), for example, reported that increasing perceptual load had a greater effect on the processing of task-irrelevant stimuli for older adults relative to younger adults. Increasing the perceptual load decreased the influence of irrelevant distractors on older adults' target identification responses, consistent with a reduction in the attentional capacity available for distractor processing.

In this version of the emotional oddball task, the standards were always squares and the distractors were always negative (sad) or neutral images. Target discrimination difficulty was varied across two blocked conditions: an easy condition, in which the target was a circle, and a hard condition, in which the target was a squoval (square-oval hybrid). Three hypotheses were of central interest. First, for the emotional valence effect of task-irrelevant distractors, we hypothesized that distractor-related neural activation would reflect resource limitations imposed by discrimination difficulty: Increased task-related attentional demands for processing targets in the hard condition would leave fewer resources available for allocation to the processing of task-irrelevant (distractor) images in visual and emotional regions, thus reducing their associated activation. Second, for target-related activation, we hypothesized that 
the hard condition would yield increased brain activation in regions associated with visual detection and attention, along with increased time required for both decisional and nondecisional components of target detection. Third, for effects of emotional valence on target detection, we hypothesized that the emotional distractors, as compared to neutral distractors, would influence the activation associated with subsequent target detection, particularly in the dorsal frontoparietal regions associated with cognitive control, as well as increase decisional and nondecisional components of RT. This latter influence of the emotional distractors should, in turn, be modulated by target discrimination difficulty and demonstrate an increased pattern of activation as target discrimination increases, due to additional monitoring, attention, and executive control required for the inhibition of the emotional distractors during the detection response.

\section{Results}

\subsection{Target detection reaction time (RT)}

For the RT and diffusion variables, we conducted analyses of variance (ANOVA) in order to investigate our hypotheses regarding the effects of target discrimination difficulty (easy vs hard) and target type (targets after negative images (TAneg) vs targets after neutral images (TAneut)). Trials on which participants failed to respond were excluded from analysis, as were trials with RTs either $<250 \mathrm{~ms}$ or $>2000 \mathrm{~ms}$ ( $<1.0 \%$ of all trials). We also excluded those 5-6 targets per target discrimination difficulty condition that did not have an intervening image following the most recent target and thus could not be assigned to either the TAneg or TAneut condition. The task difficulty effects were essentially unchanged whether the latter trials were excluded or not.

In a within subjects ANOVA of RT for all correct responses to targets, with target type and task difficulty condition as withinsubjects independent variables, the main effect of task difficulty was significant, $F(1,42)=131.92, p<0.0001, \eta_{p}^{2}=0.76$, with participants responding $128 \mathrm{~ms}(90 \%, \mathrm{CI}[46,210])$ more slowly in the hard condition ( $703 \mathrm{~ms}, \mathrm{SD}=118,90 \%, \mathrm{CI}[571,827])$ relative to the easy condition ( $575 \mathrm{~ms}, \mathrm{SD}=99,90 \%, \mathrm{CI}[463,692])$. Neither the main effect of target type nor the Target Type $\mathrm{x}$ Task Difficulty Condition interaction were significant, $F(2,42)=0.23, p=0.64$, and $F(2,42)=0.08, p=0.78$, respectively. Accuracy was $>95 \%$ in each condition, and did not vary by task difficulty condition, $\mathrm{F}(1,42)$ $=2.21, \mathrm{p}=0.14$, nor target type, $\mathrm{F}(2,42)=0.44, \mathrm{p}=0.65$ (Table 1 ).

Application of the EZ diffusion model to the RT data yielded estimates of drift rate $(v)$, nondecision time $\left(\mathrm{T}_{\mathrm{er}}\right)$, and boundary separation ( $a$ ) for each participant, in each condition. In view of the absence of any RT effects for the TAneg and TAneut conditions, we only modeled the diffusion RT data as a function of the easy and hard conditions. A within-subjects ANOVA of the diffusion model variables, with task difficulty as the independent variable and drift rate, nondecision time, and cautiousness as dependent variables, yielded significant condition effects for drift rate, $F(1,42)=22.76$, $p<0.0001, \eta_{p}^{2}=0.35$, and nondecision time, $F(1,42)=34.82$, $p<0.0001, \eta_{p}^{2}=0.45$, but not for cautiousness, $F(1,42)=1.78$,

Table 1

Target hit performance.

\begin{tabular}{lll}
\hline Condition & RT $(\mathrm{ms})$ & Accuracy \\
\hline Easy: Following Negative & $583(98)$ & $0.981(0.055)$ \\
Easy: Following Neutral & $588(105)$ & $0.985(0.034)$ \\
Hard: Following Negative & $697(126)$ & $0.965(0.059)$ \\
Hard: Following Neutral & $702(122)$ & $0.966(0.064)$ \\
\hline
\end{tabular}

Note. $n=43$. RT $=$ reaction time. Values are means, with standard deviations in parentheses. $p=0.19$. Relative to the easy condition, the hard condition was associated with a slowing of drift rate, with averages of 0.363 $(90 \% \mathrm{CI}[0.18,0.54])$ in the easy condition and $0.280(90 \%, \mathrm{CI}$ $[0.16,0.48])$ in the hard condition. Increasing discrimination difficulty also led to an increase in nondecision time, with participants averaging $0.396 \mathrm{~s}(90 \%, \mathrm{CI}[0.26,0.52])$ in the easy condition and $0.483 \mathrm{~s}(90 \%, \mathrm{CI}[0.37,0.62])$ in the hard condition (Fig. 1).

\section{2. fMRI data}

\subsubsection{Emotional valence of distractor images}

To investigate the effects of the emotional valence of distractor images in the fMRI data overall and taking target discrimination difficulty into account, we analyzed whole-brain voxelwise maps using a General Linear Model (GLM). The whole-brain voxelwise map of emotional valence of the distractor images (negative $>$ neutral), for easy and hard runs combined, yielded activation in clusters located in ventral occipital and frontal regions, as well as dorsal frontal regions (Fig. 2a; Table 2). The hard > easy contrast did not yield any significant clusters. However, for the contrast representing (easy negative $>$ easy neutral) $>$ (hard negative $>$ hard neutral), negative image-related activation was evident in the left occipital pole and right temporal-occipital fusiform cortex (Fig. 2b; Table 2).

\subsubsection{Target detection.}

To investigate the effect of target discrimination difficulty on target detection, in the same GLM, whole-brain voxelwise maps revealed a widespread effect of target-related activation, for easy and hard runs combined (Fig. 3a; Table 3). In view of the widespread activation, the threshold for this contrast was increased to $\mathrm{Z}=5$. The overall target activation yielded four clusters in the right inferior frontal gyrus, left frontal pole, left temporal fusiform cortex, and right frontal pole. The hard > easy contrast for the target-related activation yielded four clusters in the right superior parietal lobule, right inferior frontal gyrus, and right paracingulate gyrus, and left inferior frontal gyrus (Fig. 3b; Table 3). The easy > hard contrast comprised a single cluster in right superior temporal gyrus (Fig. 3c; Table 3). Analyses including nondecision time and boundary separation as continuous covariates yielded no significant clusters

\subsubsection{Emotional valence of distractors and target detection}

To examine the potential influence of the emotional valence of the distractor images on target detection, we compared activation for targets following negative images to those following neutral images in a separate GLM. As noted previously, 5-6 targets within each of the easy and hard target detection conditions did not have an intervening distractor image following the most recent target, and these trials were excluded from analysis. The TAneg > TAneut contrast, collapsing across the easy and hard runs, exhibited widespread activation. Therefore, in order to fully examine the peak regions of activation, the threshold was increased for this contrast $(Z=5)$. Clusters in the cerebellum, left middle frontal gyrus, left inferior temporal gyrus, and right precentral gyrus were significant (Fig. 4a). The hard $>$ easy contrast, representing (hard TAneg $>$ hard TAneut) $>$ (easy TAneg $>$ easy TAneut), yielded extensive activation in frontoparietal and affective related regions. Significant clusters were in the right lateral occipital cortex, bilateral inferior frontal gyrus, and right paracingulate gyrus (Fig. 4b). Activation also encompassed the anterior cingulate cortex, bilateral middle frontal gyri, bilateral parietal cortex, bilateral insula, and subcortical structures, including the right amygdala, right hippocampus, right caudate, right putamen, and thalamus (Fig. 4b; Table 4). The easy $>$ hard contrast, representing (easy TAneg $>$ easy TAneut) > 

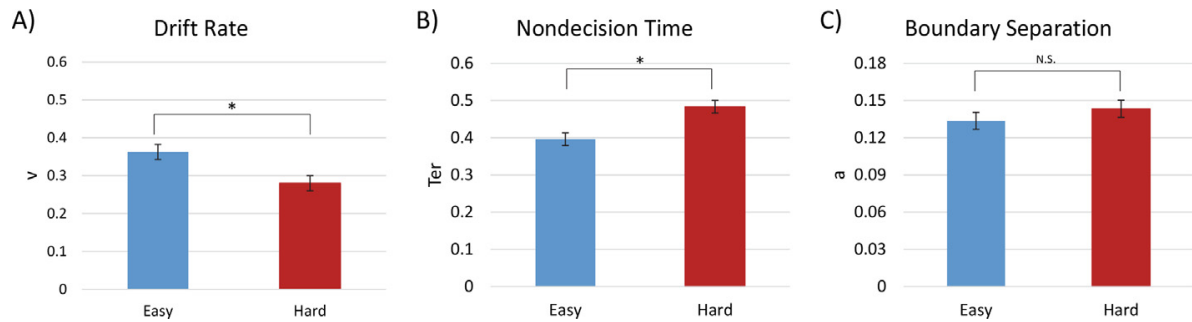

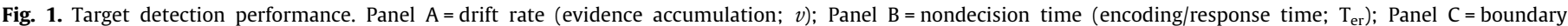
separation (cautiousness; $a$ ). Increasing target discrimination difficulty (easy vs. hard) led to a significant decrease in drift rate and increase in nondecision time.

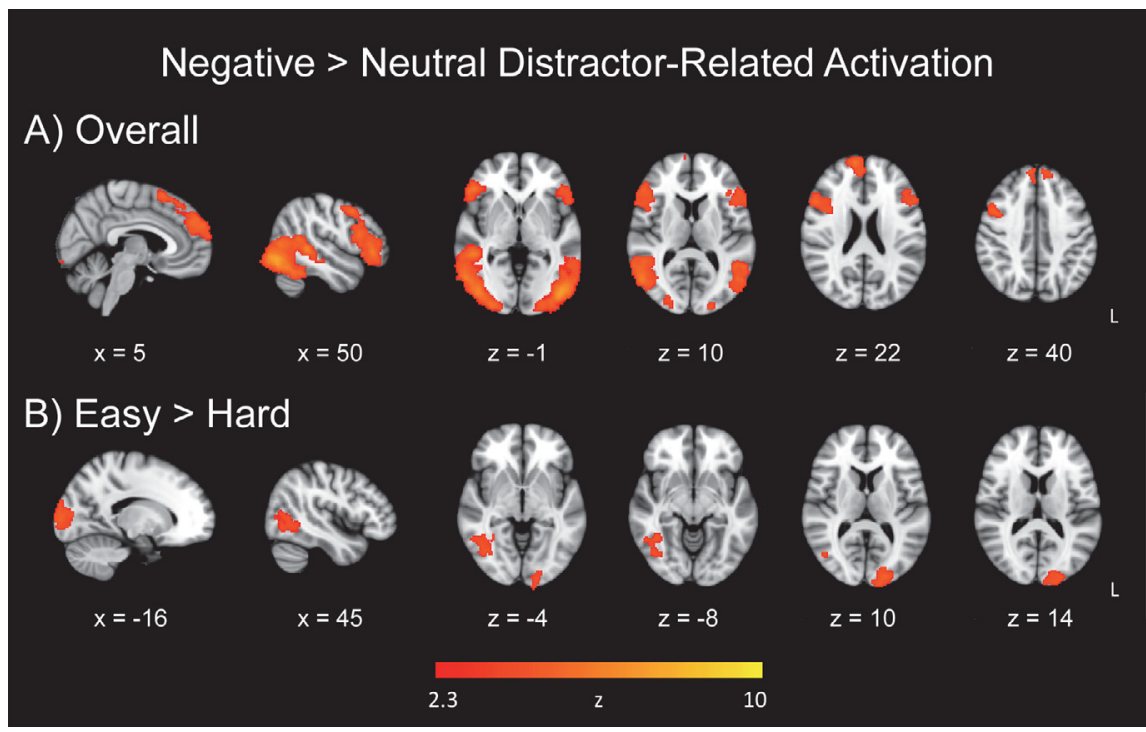

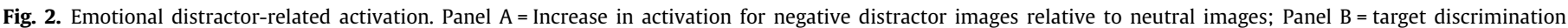
difficulty effect for negative image-related activation.

Table 2

Emotional valence effects for distractors.

\begin{tabular}{|c|c|c|c|c|c|c|}
\hline \multirow[t]{2}{*}{ Cluster } & \multirow[t]{2}{*}{ Hem } & \multicolumn{3}{|c|}{ MNI Coord (mm) } & \multirow[t]{2}{*}{ Size (voxels) } & \multirow[t]{2}{*}{$\mathrm{P}<$ value } \\
\hline & & $x$ & $y$ & $z$ & & \\
\hline \multicolumn{7}{|l|}{ Overall Negative $>$ Neutral } \\
\hline Lateral occipital cortex (inferior) & $\mathrm{R}$ & 42 & -82 & -12 & 6640 & 0.0001 \\
\hline Lateral occipital cortex (inferior) & $\mathrm{L}$ & -40 & -86 & -12 & 5930 & 0.0001 \\
\hline Inferior frontal gyrus & $\mathrm{R}$ & 50 & 32 & -2 & 2301 & 0.0001 \\
\hline Frontal pole & $\mathrm{L}$ & -48 & 40 & -10 & 1245 & 0.001 \\
\hline Frontal pole & $\mathrm{R}$ & 10 & 60 & 24 & 1014 & 0.0001 \\
\hline Superior frontal gyrus & $\mathrm{R}$ & 10 & 14 & 62 & 554 & 0.05 \\
\hline \multicolumn{7}{|l|}{ Easy $>$ Hard } \\
\hline Occipital Pole & $\mathrm{L}$ & -20 & -94 & 10 & 783 & 0.01 \\
\hline Temporal-occipital fusiform cortex & $\mathrm{R}$ & 40 & -52 & -6 & 512 & 0.05 \\
\hline
\end{tabular}

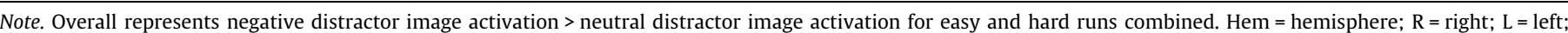

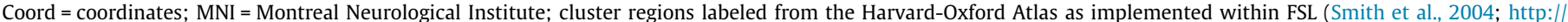
www.fmrib.ox.ac.uk/fsl). Clusters are reported from highest to lowest Z value for each contrast. There were no significant clusters for the Hard > Easy contrast.

(hard TAneg $>$ hard TAneut), reflected increased activation centered in the left occipital pole (Fig. 4c)

To investigate covariations between the diffusion variables and fMRI data, three separate one-sample t-tests with covariates of interest were conducted for drift rate, nondecision time, and boundary separation for each GLM. Maps including drift rate as a continuous covariate in the hard > easy contrast, for TAneg > TAneut, yielded significant clusters of activation in the right superior parietal lobule/postcentral gyrus, right inferior temporal gyrus, left middle temporal gyrus, and left supramarginal gyrus which decreased as a function of increased drift rate (Fig. 5; Table 5).
Therefore, in these regions, for task difficulty related activation (hard > easy contrast) for targets following negative images, increasing activation was associated with lower drift rate, reflecting slower decisional processing. Analyses including nondecision time and boundary separation as continuous covariates yielded no significant clusters.

To demonstrate the inverse relation between drift rate and fMRI data, we chose the cluster peaks from the contrast of interest, (hard TAneg $>$ hard TAneut) $>$ (easy TAneut $>$ easy TAneut), shown in Table 5 , and created a region of interest (ROI) with an $8 \mathrm{~mm}$ diameter centered on each peak. From the participant-level models, we 


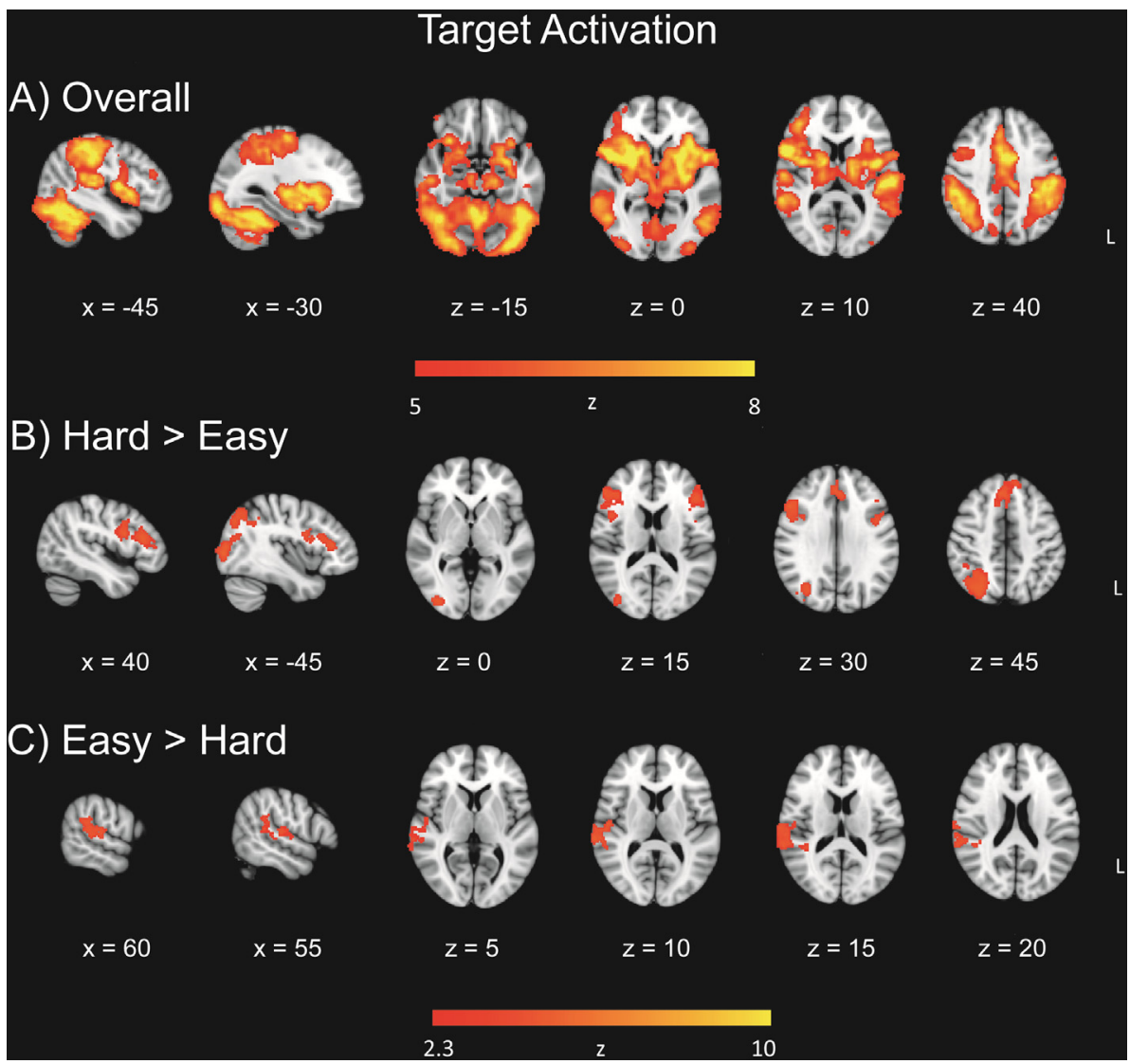

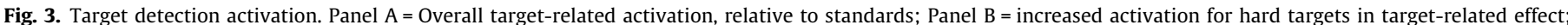
Panel C = increased activation for easy targets in target-related effect.

Table 3

Target detection effects.

\begin{tabular}{|c|c|c|c|c|c|c|}
\hline \multirow[t]{2}{*}{ Cluster } & \multirow[t]{2}{*}{ Hem } & \multicolumn{3}{|c|}{ MNI Coord (mm) } & \multirow[t]{2}{*}{ Size (voxels) } & \multirow[t]{2}{*}{$\mathrm{P}<$ value } \\
\hline & & $x$ & $y$ & $z$ & & \\
\hline \multicolumn{7}{|l|}{ Overall Target $>$ Baseline } \\
\hline Inferior frontal gyrus & $\mathrm{R}$ & 52 & 30 & 10 & 82,735 & 0.0001 \\
\hline Frontal pole & $\mathrm{L}$ & -44 & 44 & 14 & 250 & 0.0001 \\
\hline Temporal fusiform cortex & $\mathrm{L}$ & -32 & -6 & -36 & 16 & 0.001 \\
\hline Frontal pole & $\mathrm{R}$ & 20 & 66 & -12 & 14 & 0.001 \\
\hline \multicolumn{7}{|l|}{ Hard > Easy } \\
\hline Superior parietal lobule & $\mathrm{R}$ & 28 & -70 & 42 & 1613 & 0.0001 \\
\hline Inferior frontal gyrus & $\mathrm{R}$ & 46 & 22 & 22 & 1297 & 0.0001 \\
\hline Paracingulate gyrus & $\mathrm{R}$ & 2 & 30 & 38 & 920 & 0.001 \\
\hline Inferior frontal gyrus & $\mathrm{L}$ & -46 & 28 & 18 & 633 & 0.01 \\
\hline \multicolumn{7}{|l|}{ Easy $>$ Hard } \\
\hline Superior temporal gyrus (posterior) & $\mathrm{R}$ & 68 & -30 & 10 & 890 & 0.001 \\
\hline
\end{tabular}

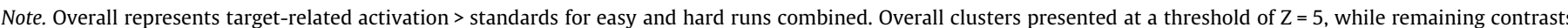

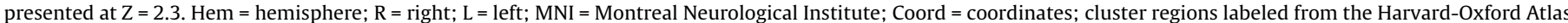
as implemented within FSL (Smith et al., 2004; http://www.fmrib.ox.ac.uk/fsl). Clusters are reported from highest to lowest $Z$ value for each contrast.

used FSL Featquery to extract parameter estimates for the contrast of interest, across all voxels within each of the ROIs. The parameter estimates were intensity normalized, converted to percent signal change, and averaged within each ROI for each participant. From the group level covariate model, drift rate correlated with percent signal change in the right superior lobule/postcentral gyrus, $\mathrm{r}=-0.52, \mathrm{p}=0.0003(95 \%, \mathrm{CI}[-0.71,-0.26])$ (Fig. 6, Panel A), the right inferior temporal gyrus, $r=-0.49, p=0.0009(95 \%, C I$ $[-0.68,-0.21]$ ) (Fig. 6, Panel B), the left middle temporal gyrus, $\mathrm{r}=-0.59, \mathrm{p}<0.0001(95 \%, \mathrm{CI}[-0.75,-0.34])$ (Fig. 6, Panel C), and the left supramarginal gyrus, $\mathrm{r}=-0.54, \mathrm{p}=0.0002(95 \%, \mathrm{CI}[-0.72$, $-0.28]$ ) (Fig. 6, Panel D). These plots were constructed for data visualization purposes and include confidence intervals in order to demonstrate the relationship between drift rate and significant clusters from the contrast.

\section{Discussion}

\subsection{Emotional valence effects}

Our first hypothesis predicted that target discrimination difficulty would modulate emotional processing in the brain due to the reallocation of attentional resources, specifically a reduced 


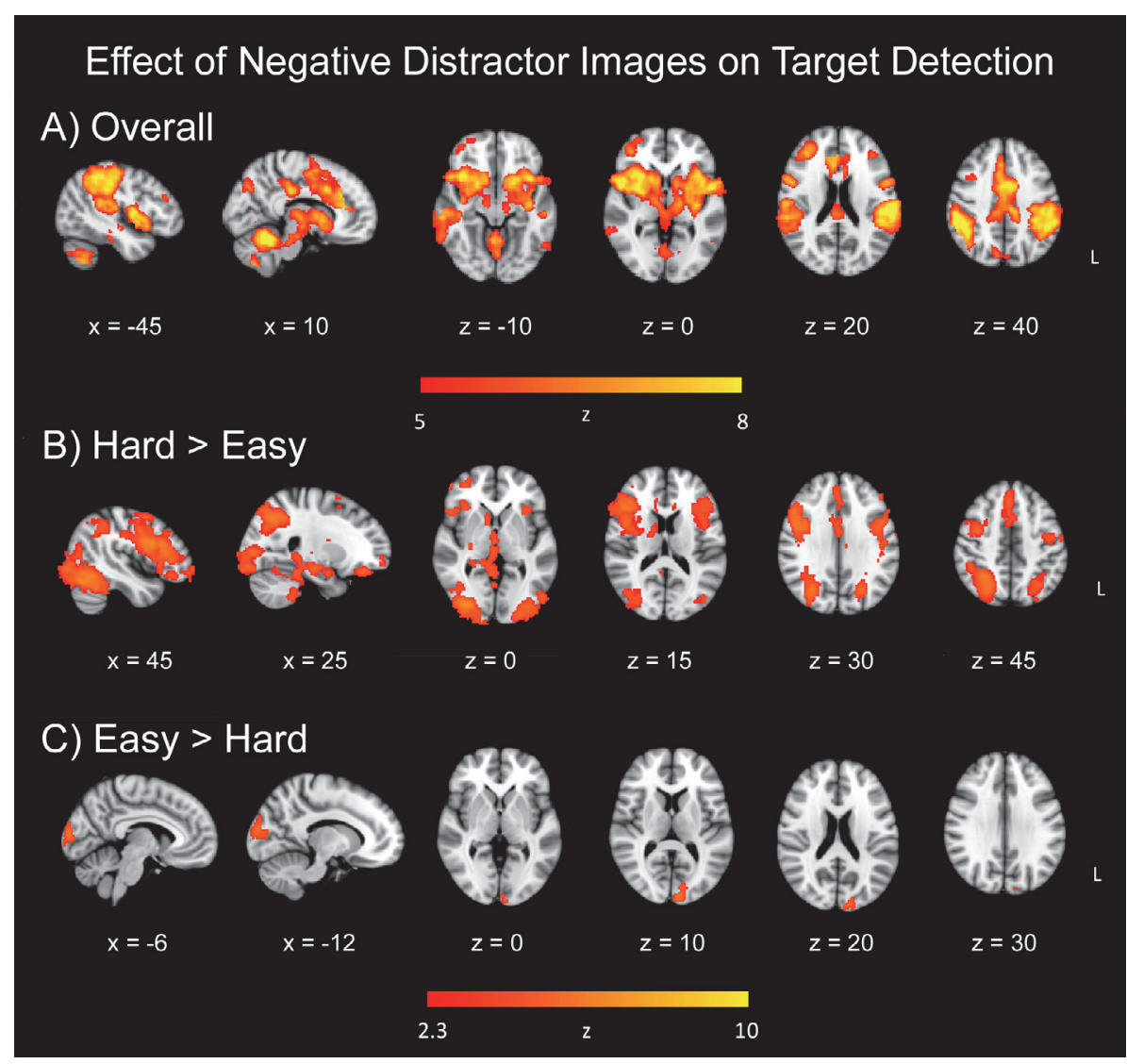

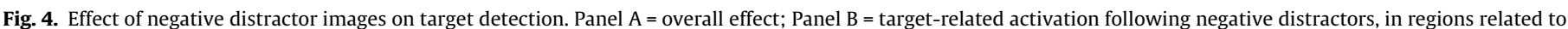

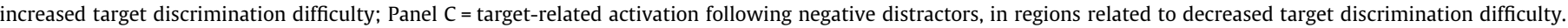

Table 4

Effects of preceding negative distractor images on target detection.

\begin{tabular}{|c|c|c|c|c|c|c|}
\hline \multirow[t]{2}{*}{ Cluster } & \multirow[t]{2}{*}{ Hem } & \multicolumn{3}{|c|}{ MNI Coord (mm) } & \multirow[t]{2}{*}{ Size (voxels) } & \multirow[t]{2}{*}{$\mathrm{P}<$ value } \\
\hline & & $x$ & $y$ & $z$ & & \\
\hline \multicolumn{7}{|c|}{ Overall Targets Following Negative $>$ Neutral } \\
\hline Middle frontal gyrus & $\mathrm{L}$ & -38 & 34 & 26 & 356 & 0.0001 \\
\hline Inferior temporal gyrus & $\mathrm{L}$ & -60 & -52 & -24 & 287 & 0.0001 \\
\hline Precentral gyrus & $\mathrm{R}$ & 32 & -10 & 58 & 100 & 0.0001 \\
\hline \multicolumn{7}{|l|}{ Hard $>$ Easy } \\
\hline Lateral occipital cortex (inferior) & $\mathrm{R}$ & 32 & -84 & -2 & 18,120 & 0.0001 \\
\hline Inferior frontal gyrus & $\mathrm{R}$ & 50 & 14 & 26 & 5467 & 0.0001 \\
\hline Inferior frontal gyrus & $\mathrm{L}$ & -44 & 8 & 24 & 3515 & 0.0001 \\
\hline Paracingulate gyrus & $\mathrm{R}$ & 2 & 32 & 38 & 1677 & 0.0001 \\
\hline \multicolumn{7}{|l|}{ Easy $>$ Hard } \\
\hline Occipital pole & $\mathrm{L}$ & -8 & -96 & 12 & 441 & 0.05 \\
\hline
\end{tabular}

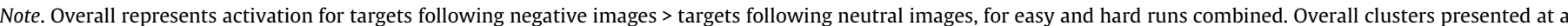

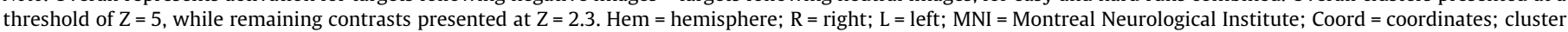

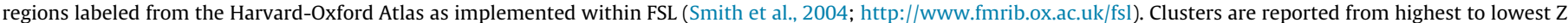
value for each contrast.

level of activation to emotional distractors within a difficult discrimination condition relative to an easier discrimination. While some studies have shown that emotion is processed irrespective of attentional capacity and available processing resources (Anderson et al., 2003; Morris et al., 1999), our hypothesis was supported by the variation in the emotional valence effect (greater activation for sad images relative to neutral images) as a function of task difficulty: There was no significant activation in the hard > easy contrast, whereas the easy $>$ hard contrast revealed occipital activation (Fig. 2, Panel B). Similar to other fMRI and ERP studies, our results suggest that emotional distractors, sad ones in this case, are processed when sufficient attentional resources are available in certain tasks, providing evidence for an interactive relation between the emotionally salient distractors and top-down attentional control, when attentional demands are lower (Blair et al., 2007; Doallo et al., 2006; Lange et al., 2003; Maki-Marttunen et al., 2014; Pessoa et al., 2002a,b; Shafer et al., 2012). It should be noted that our finding may be limited to our particular emotional oddball task, with the use of sad images depicting sociallyrelevant scenes, whereas different categorizations of emotion, such 


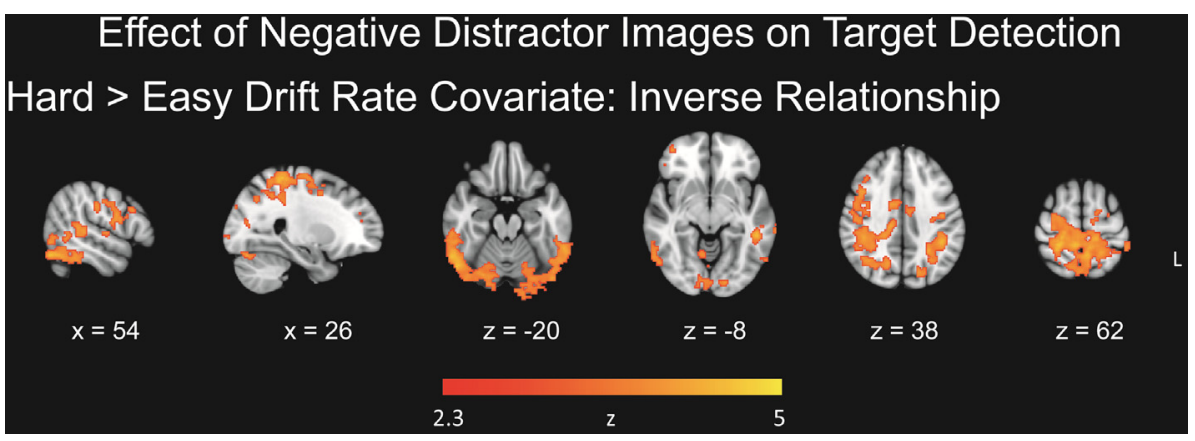

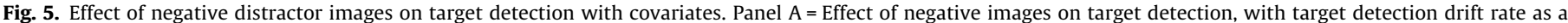
covariate. The effect of the preceding negative images within the hard > easy activation was correlated negatively with target detection drift rate.

Table 5

Effects of preceding negative distractor images on target detection, with covariate.

\begin{tabular}{|c|c|c|c|c|c|c|}
\hline \multirow[t]{2}{*}{ Cluster } & \multirow[t]{2}{*}{ Hem } & \multicolumn{3}{|c|}{ MNI Coord (mm) } & \multirow[t]{2}{*}{ Size (voxels) } & \multirow[t]{2}{*}{$\mathrm{P}<$ value } \\
\hline & & $x$ & $y$ & $z$ & & \\
\hline \multicolumn{7}{|c|}{ Hard > Easy Drift Rate Covariate: Inverse Relationship } \\
\hline Superior parietal lobule/postcentral gyrus & $\mathrm{R}$ & 26 & -38 & 62 & 9528 & 0.0001 \\
\hline Inferior temporal gyrus & $\mathrm{R}$ & 60 & -54 & -12 & 2741 & 0.0001 \\
\hline Middle temporal gyrus & $\mathrm{L}$ & -50 & -40 & -8 & 1867 & 0.0001 \\
\hline Supramarginal gyrus & $\mathrm{L}$ & -42 & -42 & 38 & 1387 & 0.0001 \\
\hline
\end{tabular}

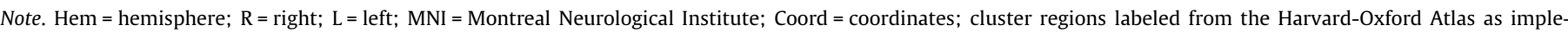
mented within FSL (Smith et al., 2004; http://www.fmrib.ox.ac.uk/fsl). Clusters are reported from highest to lowest $\mathrm{Z}$ value for each contrast.

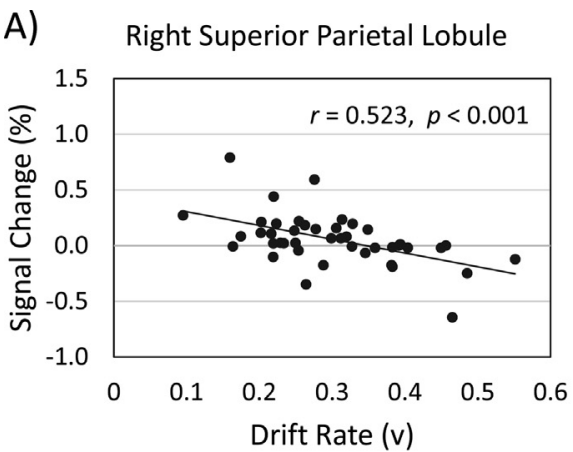

C)

Left Middle Temporal Gyrus

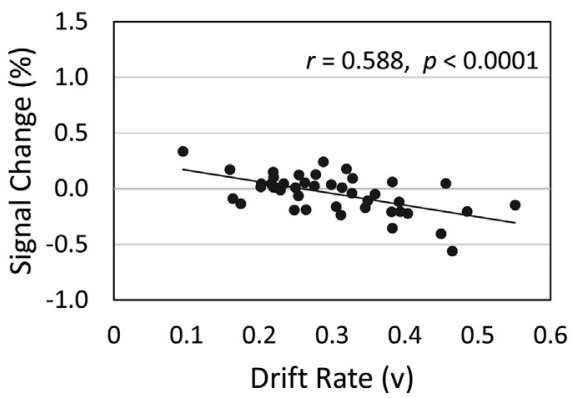

B) Right Inferior Temporal Gyrus

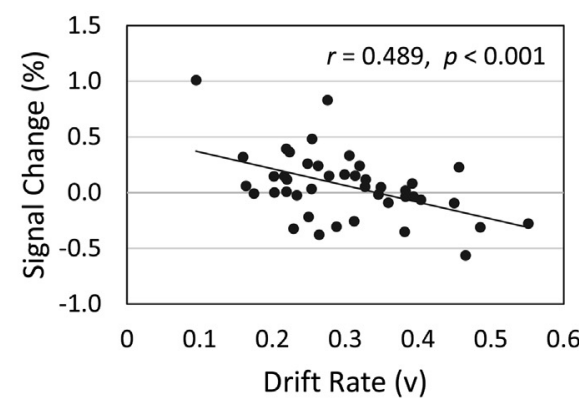

D)

Left Supramarginal Gyrus

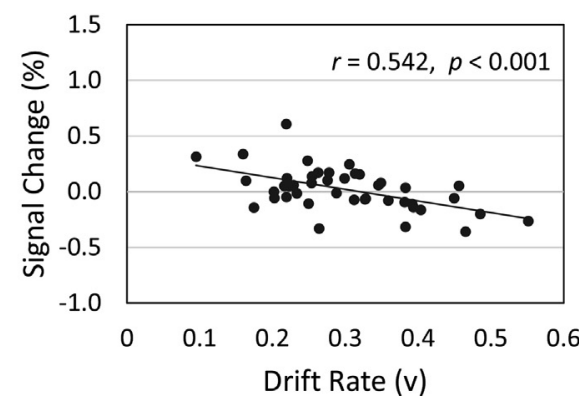

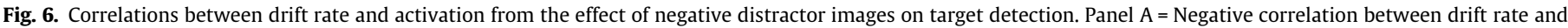

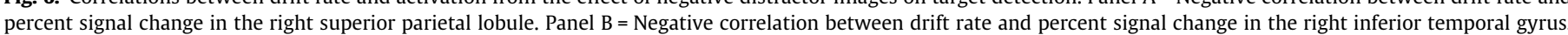

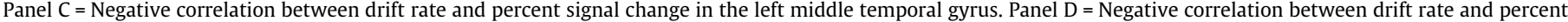
signal change in the left supramarginal gyrus.

as threatening images or depictions of fear, may yield disparate results, or there may be differences in emotional stimuli's effect on global or local target detection (Hartikainen et al., 2010, 2012; Schmidt et al., 2015).
For the easy and hard runs combined, activation associated with the negative distractor images, relative to neutral images, was evident in ventral occipital and frontal regions, and dorsal frontal regions (Fig. 2, Panel A). Activation in lateral occipital cortex 
suggests that participants engaged in more visual processing of negative, as compared to neutral, images. Inferior frontal regions have been implicated previously in processing of infrequent stimuli, response inhibition, processing informational content of distractors with emotional content, as well as executing control over emotional distraction in patients with depression (Wang et al., 2008c). In our emotional oddball task, sad stimuli were processed in both dorsal and ventral frontal regions, particularly the frontal pole bilaterally, and the right inferior frontal and right superior frontal gyri, highlighting the relevance of cortical regions in emotion processing in addition to subcortical regions (Dolcos et al., 2011a,b; Kober et al., 2008; Mather and Carstensen, 2003).

It is interesting to note that there was no significant activation in our voxelwise emotion effect contrasts in traditional subcortical structures known to process emotional stimuli, particularly the amygdala. However, previous neuroimaging studies of healthy participants have also reported that amygdala activation is absent when sad images are compared to neutral images (Blair et al., 1999; Kesler-West et al., 2001; Phillips et al., 1997). Studies suggest that amygdala activation is only enhanced when emotion is task relevant (Gur et al., 2002; Hariri et al., 2000), or is only preferentially activated to sad distractors after negative mood induction (Wang et al., 2006) (but cf. Ritchey et al., 2008). Upon investigation of the unthresholded contrast, this activation is indeed present, but simply not strong enough to elicit activation passing threshold when compared to other task-irrelevant images. Also of note, the amygdala is mainly associated with fear response, and perhaps our use of sad stimuli, of lower valence and arousal as compared to fearful stimuli, did not elicit as strong activation.

\subsection{Discrimination difficulty effects}

Consistent with our second hypothesis, the behavioral data in the hard condition yielded higher mean RTs, as well as the component processes of drift rate $(v)$, and nondecision time $\left(\mathrm{T}_{\mathrm{er}}\right)$, relative to the easy condition, indicating that discrimination difficulty affected both decisional and nondecisional components of RT (Fig. 1). This finding provides behavioral evidence that target discrimination difficulty modulates both bottom-up encoding and the motor response, as well as top-down decisional processing. Interestingly, the cautiousness variable $(a)$ did not vary significantly across the task difficulty conditions, implying that the increased discrimination difficulty slowed the information accumulation required for a decision, and associated encoding and response processes, but did not change the amount of information required for a response.

The fMRI data also indicated a significant effect of discrimination difficulty: Participants exhibited more widespread activation to targets in the hard (vs. easy) condition, with clusters centered in the right superior parietal lobule, bilateral inferior frontal gyri, and right paracingulate gyrus, which have all been tied to the attentional and/or salience networks (Fig. 3, Panel B). The superior parietal lobule has been previously linked to the dorsal attention network involved in top-down visuospatial orienting and has also been shown to be modulated by task difficulty and informational load (Cusack et al., 2010; Petersen and Posner, 2012; Ritchey et al., 2008; Tomasi et al., 2007). The inferior frontal gyrus is commonly activated during stimulus-driven attention, particularly infrequent stimuli, and has been shown to be altered with task difficulty (Chikazoe et al., 2009; Tomasi et al., 2007). The paracingulate gyrus was increasingly active for hard targets, indicating its functional similarity to the dorsal anterior cingulate and involvement in top-down attentional control (Touroutoglou et al., 2012; Vincent et al., 2008). In our task, these regions appear to support successful target processing and/or response when task demands are high, perhaps via attention to task-relevant salient stimuli, and/or dorsal frontoparietal top-down orienting.

\subsection{Influence of emotional distractors on subsequent target detection}

Our third hypothesis that emotional valence modulates attentional processing was supported in the brain data, but not behavioral RT measures. As predicted, there was significantly more activation to targets following negative images than targets following neutral images, suggesting an influence of the sad distractors on brain regions supporting target detection (Fig. 4, Panel A). Interestingly, this difference in activation was not mirrored in the behavioral data. There was no significant difference in either mean RT, diffusion variables, or accuracy for targets following negative images relative to those following neutral images nor differences between task difficulty conditions. Contrary to our hypothesis, this result suggests that the emotional valence of sad images preceding targets does not affect either decisional or nondecisional components of RT in our paradigm. In another go/no-go task involving threat-related stimuli (vs emotionally neutral stimuli), which have a high biological relevance, similarly had no effect on RT, but did interfere with response inhibition (Hartikainen et al., 2012). Nonetheless, we expected that RTs would increase for targets following sad images as compared to neutral, as disruption of task performance after exposure to emotional distractors has been previously reported (Blair et al., 2007; Hartikainen et al., 2000). Since this study contained healthy individuals, perhaps the difference in activation is what permitted statistically equivalent behavioral performance: Brain regions differentially active for top-down control over emotional distraction were able to facilitate successful performance, presumably reflecting capable executive control and realignment of attention to task-relevant stimuli in the face of emotional distraction.

Contrasts of target discrimination difficulty (hard > easy; easy $>$ hard), for the effects of preceding negative distractor images on target detection, demonstrated significant clusters. The hard > easy contrast (Fig. 4, Panel B) revealed extensive occipital cortex activation, demonstrating increased visual processing for targets following sad images. Activation in the inferior frontal gyrus, bilaterally, was also evident in this contrast. The left inferior frontal gyrus has been found to be related to effortful control of the impact of emotional distraction on goal-oriented behavior (Dolcos et al., 2006). It was also found that depressed patients demonstrated increased activation in the right inferior frontal gyrus for targets after negative images, compared to controls (Wang et al., 2008c). The authors postulated that altered right inferior frontal gyrus activation in depression represented altered executive control over emotional distraction. In the present study, we demonstrate that, within a sample of healthy older adults, both the left and right inferior frontal gyri demonstrate increased activation to targets following sad images, as compared to targets following neutral images, only when the task is more difficult. Therefore these regions are also modulated by target discrimination difficulty in the present emotional oddball task. This result provides potential support for the inferior frontal gyri's proposed role in control over emotional distraction, since in the more difficult task, increased bilateral inferior frontal gyrus activation was observed, perhaps reflecting increased control over emotional distraction required with increased task difficulty.

Wang et al. (2008c) previously found that healthy controls, as compared to depressed patients, demonstrated stronger activation to targets after sad images in the insula and dorsal anterior cingulate cortex (dACC), similar to our results. In addition, Wang et al. found that rostral anterior cingulate cortex (rACC) had stronger activation for targets after sad images relative to targets after neutral images, in healthy controls, but not depressed patients. 
The rACC has been linked to affective processing, while dACC is thought to execute cognitive processing and top-down control, and both subdivisions have been proposed as potential targets for depressive treatment (Alexopoulos et al., 2012; Pizzagalli, 2011; Weissman et al., 2005). Similarly, we found that in this sample of healthy older adults, the rACC was differentially active for targets following sad images, but it is interesting to note that we found the dACC to show differential activation to targets following sad images as well (Fig. 4, Panel A). Our results extend Wang et al.'s emotional control effect hypothesis by demonstrating that both subdivisions of the ACC are differentially engaged depending on task difficulty. This difference yielded stronger activation in the hard > easy contrast, with the peak cluster in the paracingulate gyrus, indicating that the cingulate is indeed implicated in topdown attentional constraints on emotional processing and processing of social stimuli (Gu and Han, 2007; Touroutoglou et al., 2012; Vincent et al., 2008; Walter et al., 2004).

For the hard > easy contrast, the middle frontal gyrus, parietal cortex, and insula demonstrated activation to targets, confirming their involvement in attentional and target-related processing (Dosenbach et al., 2006, 2007; Eckert et al., 2009; Wang et al., 2008c). Other regions of particular interest from the hard > easy contrast of emotional control included subcortical affective structures. The amygdala, hippocampus, caudate, putamen, and thalamus, predominantly in the right hemisphere, demonstrated significant activation to task-relevant targets following taskirrelevant sad emotional distractors. It is possible that these regions demonstrate increased activation in order for participants to either successfully disengage from emotional distraction, and/ or execute top-down attention control in order to reorient attention to task-relevant targets (Gu and Han, 2007; Vincent et al., 2008). Yet, this may also suggest delayed processing of emotional distractors that is exacerbated in the hard condition.

Despite the potential delayed effect from previously presented emotional distractors, the cortico-limbic system is indeed involved in the emotional response and resulting adaptive behavior. Increased anterior cingulate cortex activation has been linked to top-down resolution of emotional conflict, as well as increased functional connectivity with the amygdala in an emotion processing task (Comte et al., 2016). Furthermore, there has been fMRI evidence that a frontoparietal control system exists that is spatially, and potentially functionally, interposed between components of the dorsal attention (task-positive, top-down influence over externally oriented tasks) and hippocampal-cortical memory systems (task-negative, internally oriented). This control system can show sustained activity throughout a task block, perhaps due to constant information integration or sustained task set maintenance. It is also recruited when judgments are uncertain, and is implicated in updating and implementing goal-directed behavior, which in the present study, could have been exacerbated in the hard condition (Dosenbach et al., 2006; Velanova et al., 2003; Vincent et al., 2008; Yarkoni et al., 2005). The caudate has been shown to be a part of the frontoparietal control system, and is often engaged by tasks requiring controlled information processing, in addition to its implications in visual tasks, along with the putamen and thalamus (Herath et al., 2001; Vincent et al., 2008). The hippocampal formation has been shown to be strongly correlated with ventromedial prefrontal cortex, dorsal frontal cortex, lateral temporal cortex, posterior cingulate cortex, and ventral posterior inferior parietal lobule, and the frontoparietal control system is adjacent to systems functionally correlated with the hippocampal formation, which may be helpful in tasks that utilize past experience to make decisions (Vincent et al., 2008).

The relation between the effect of negative images on the subsequent targets exhibited a complex relation with target discrimination difficulty, such that the effect of the preceding negative images within the hard > easy activation was correlated negatively with target detection drift rate. That is, increasing activation in those regions exhibiting an interactive effect of task difficulty and preceding negative images (parietal and temporal regions) exhibited slower information accumulation rates. This result is supported by our task difficulty effect seen in drift rate, demonstrating how a more difficult task results in slower information assessment and thus compromises the quality of the central decision process (Fig. 1). Whereas both drift rate and nondecision time varied with task difficulty in behavior, only drift rate exhibited a relation to target detection activation. This finding provides evidence for alterations in the decisional, or top-down, component of RT in relation to the superior parietal lobule, temporal gyrus, and supramarginal gyrus activation to targets following emotional distraction.

\subsection{Limitations}

Our study has several limitations. Here, we included only older adults, in an attempt to maximize the potential effects of attentional load, but to investigate age-related effects either a younger comparison group or a wider age range is needed. Therefore, in the present study, the results represent general emotioncognition interactions, when one component of the system is more heavily taxed, target discrimination in this case, and do not lead to any conclusions about aging specifically. In addition, while the emotional oddball task has the advantage of separating the distractor and target events in time, the target is by definition a rare event. As a result, there were relatively few trials in the relevant target-distractor combinations (10-16 trials), thus limiting the statistical power for both the fMRI and behavioral effects. It would be beneficial for future studies increase the number of trials, to obtain more fine-grained estimates of the duration of the distraction associated with the negative images. One limitation of a mixed design with blocked conditions is that it can only provide information about relative differences between our target discrimination difficulty conditions. Future research could utilize a purely eventrelated design in order to examine differences in easy and hard visual discrimination with a singular baseline and rule out any potential differences in easy > hard activation, as reflected in our negative $>$ neutral contrast, as a consequence of a sustained increase in attention resulting in the hard condition, leading to an elevated shift in the baseline.

\subsection{Conclusions}

Overall, our results demonstrate an influence of target discrimination difficulty on neural and behavioral correlates of emotioncognition interactions in healthy older adults, and provide support for theories regarding the contributions of both automatic and topdown processes to the regulation of emotional distraction (Pessoa et al., 2002a,b; Shafer et al., 2012). Activation associated with taskirrelevant emotional distractors was apparent in both dorsal and ventral cortical regions, specifically inferior frontal gyri and the cingulate, and this distractor-related activation was attenuated by increasing target discrimination difficulty. Increasing target discrimination difficulty also led to increased target-related activation in frontoparietal regions. Task-irrelevant negative distractor images affected the activation associated with subsequent target detection, particularly in regions associated with target discrimination difficulty, expressed as an enhancement of task difficultyrelated activation for targets following negative distractors. At the participant level, this influence of the sad distractors, on subsequent target-related activation, was also associated with slower accumulation of target-related information. These findings indicate that emotion-cognition interactions, in the emotional oddball 
task, vary in relation to available attentional resources, and that these interactions have an influence on target detection beyond the duration of the distracting sad event.

\section{Methods and materials}

\subsection{Participants}

The final sample comprised 43 participants (25 women; 40 right-handed) $59-81$ years of age $(M=68.3, S D=6.2$ years). Participant characteristics, including handedness and Geriatric Depression Scale measures, are provided in Table 6 (Lesher and Berryhill, 1994; Oldfield, 1971). All participants were free of significant health problems (including atherosclerosis, neurological and psychiatric disorders), and were not taking medications known to affect cognitive function or cerebral blood flow (except antihypertensive agents). Participants gave written informed consent for a protocol approved by the Duke University Institutional Review Board. Six participants were excluded; three due to Mini-Mental State Examination (MMSE; Folstein et al., 1975) score less than 27 , and three due to poor performance on the oddball task during scanning ( $<75 \%$ accuracy in either of the task conditions).

\subsection{Emotional oddball task}

While in the scanner, participants performed a modified version of a go/no-go emotional oddball task (Wang et al., 2005, 2006, 2008a,b,c). Participants viewed a series of displays and made a button-press detection response only at the occurrence of an infrequent oddball target, in the context of infrequent distractors and more frequent, standard items (squares). Distractors were images either negative or neutral in valence used previously by Wang et al. (2005). Negative images were centered on themes of despair, grief, interment, incarceration, and poverty, and had average ratings of mildly sad, or sad from a pilot behavioral study, and neutral images were chosen for inclusion according to ratings of emotional neutrality (Wang et al., 2005). No response to the distractor images was required. Differences in visual complexity were qualitatively controlled; emotionally negative images (e.g., people portraying sad expressions) and neutral images (e.g., people shopping) were closely matched in the number of people per scene, postural features, gaze direction, and gender, with the number of distinguishable faces restricted to fewer than six per image, and did not differ in visual complexity across target discrimination difficulty block. The task included two levels of difficulty (easy and hard), in which the visual discrimination of targets from the standards varied across scanner runs. In the easy condition, the targets were circles and thus easily discriminable from the standard squares (Fig. 7, Panel A). In the hard condition, the targets were squovals (i.e., squares with rounded corners) that were more difficult to discriminate from standards (Fig. 7, Panel B).

Each run comprised 126 trials, which consisted of a randomized sequence of 98 standards ( $78 \%$ of trials), 10 neutral distractors ( $8 \%$

Table 6

Participant characteristics.

\begin{tabular}{ll}
\hline & Mean \\
\hline Education (years) & $17.0(2.0)$ \\
MMSE & $29.0(1.0)$ \\
Edinburgh Inventory & $81.50(32.12)$ \\
GDS-Short Form & $0.73(1.10)$
\end{tabular}

Note. $n=43$. Values are means, with standard deviations in parentheses. MMSE = Mini-mental state exam (Folstein et al., 1975); Edinburgh inventory = handedness scale (Oldfield, 1971). GDS = Geriatric Depression Scale (Lesher and Berryhill, 1994). of trials), 10 negative distractors ( $8 \%$ of trials), and 8 targets (6\% of trials). Participants performed eight runs of the oddball task: four easy runs and four hard runs. One of two different run orders with pseudorandomized run conditions were randomly assigned to each participant in order to control for run condition order effects. Across all runs, there were 64 target trials total, with 32 target trials in each of the easy and hard conditions. In the easy condition, there were ten targets following negative images, 16 targets following neutral images, and six targets following neither image type (i.e., no distractor occurred subsequent to the most recent target). In the hard condition, there were 14 targets following negative images, 13 targets following neutral images, and five targets following neither image type.

The use of a mixed design allowed for the maximization of the distinction between event-related stimuli, the easy and hard targets (circles and squovals) and images, and target discrimination difficulty, which can be achieved most effectively via presentation in different blocks. Second, we wanted to be sensitive to any potential differences in cautiousness, or the amount of information required for a decision (described in further detail in Section 4.3. Reaction Time Analyses), between the easy and hard targets. To achieve this we estimated the boundary separation parameter (a) in the diffusion reaction time model, which represents cautiousness. Estimating this parameter required a blocked design, because the model assumes that cautiousness is set by the observer over a series of trials and cannot be set specifically on a trial-by-trial basis.

To avoid habituation effects, the targets varied in size and color (Wang et al., 2005). Each display was presented for 1500 ms; interstimulus duration was $2000 \mathrm{~ms}$; and two to five standards (squares) intervened between infrequent stimuli (targets or images), ranging from $9 \mathrm{~s}$ to $19.5 \mathrm{~s}$ apart. The standards varied in size and color, and images were also presented in color (Fig. 7). Participants held a fiber optic response box (Current Designs, Philadelphia, PA, USA) with the right hand and were instructed to make a button press response using their right index finger upon detection of a target shape (circle or squoval). Reaction time was measured from target onset, and visual display presentation and response recording were controlled by CIGAL (Voyvodic, 1999).

\subsection{Reaction time analyses}

In addition to analyses of mean RT for target hits, we used a version of Ratcliff's diffusion model to characterize different components of the decision process in RT (Ratcliff, 1978; Ratcliff et al., 2016). These include the rate of information accumulation towards a decision boundary (drift rate; $v$ ), the visual processing and motor response (nondecision time; $\mathrm{T}_{\mathrm{er}}$ ), and response cautiousness (boundary separation; $a$ ). Whereas the original model contains additional parameters and was developed to describe two-choice RT, it has been extended to go/no-go tasks as used here (Gomez et al., 2007). We employed a simplified version of Ratcliff's model, the EZ Diffusion Model (Wagenmakers et al., 2007, 2008), which uses overall RT mean, accuracy, and variance for each participant to estimate the drift rate, nondecision time, and cautiousness components of the detection response. This modification of the original model is appropriate for data with relatively few trials per condition and high accuracy, as in the present experiment (van Ravenzwaaij and Oberauer, 2009; Voss et al., 2013; Wagenmakers et al., 2007).

\subsection{MRI data acquisition}

We conducted MRI scanning on a 3.0 T GE MR750 whole-body $60 \mathrm{~cm}$ bore MRI scanner (GE Healthcare, Waukesha, WI) equipped with $50 \mathrm{mT} / \mathrm{m}$ gradients and a $200 \mathrm{~T} / \mathrm{m} / \mathrm{s}$ slew rate. An eight- 


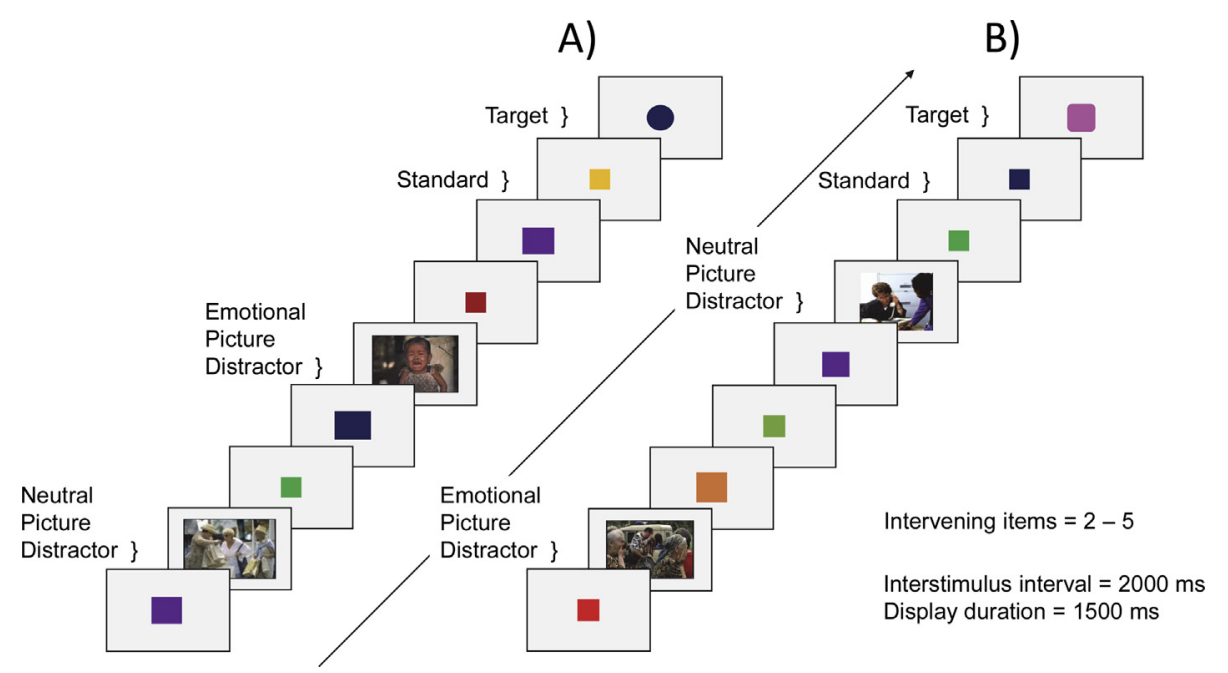

Fig. 7. Emotional oddball task. Panel $A=$ easy condition, in which the target is a circle; Panel $B=$ difficult condition, in which target is square-oval hybrid (squoval).

channel head coil was used for radio frequency reception. Participants wore earplugs to reduce scanner noise and foam pads surrounded the head to reduce head motion. Imaging began with 3-plane (straight axial/coronal/sagittal) localizer FSE images that defined a volume for data collection. A semi-automated high-order shimming program ensured global field homogeneity. We acquired one run of T1-weighted anatomical imaging, and one run of resting-state (eyes open), followed by eight runs of event-related, T2 $*$-weighted imaging.

The T1-weighted anatomical images were 162 straight axial slices acquired with a 3D fast inverse-recovery-prepared spoiled gradient recalled $(\mathrm{SPGR})$ sequence $(\mathrm{TR}=7.644 \mathrm{~ms}$, echo time [TE] $=2.936 \mathrm{~ms}$, inversion recovery time $[\mathrm{TI}]=450 \mathrm{~ms}$, field of view $[\mathrm{FOV}]=256 \mathrm{~mm}$, flip angle $=12^{\circ}$, voxel size $=1 \times 1 \times 1 \mathrm{~mm}$, $256 \times 256$ matrix, and a sensitivity encoding [SENSE] factor of 2 ) using an array spatial sensitivity encoding technique and extended dynamic range. Event-related functional imaging included 36 contiguous slices acquired at an axial oblique orientation, parallel to the AC-PC plane $(\mathrm{TR}=2 \mathrm{~s}, \mathrm{TE}=25 \mathrm{~ms}, \quad \mathrm{FOV}=256 \mathrm{~mm}$, flip angle $=90^{\circ}$, voxel size $=4 \times 4 \times 4 \mathrm{~mm}, 64 \times 64$ matrix, and a SENSE factor of 1). For each event-related run, 126 brain volumes were collected.

\section{5. fMRI data analyses}

\subsubsection{Preprocessing pipeline}

The structural brain images were skull-stripped using the FSL brain extraction tool (Smith, 2002). Data quality was assessed using a quality assurance tool that quantifies several metrics including Signal-to-Noise (SNR), Signal-Fluctuation-to-Noise (SFNR), motion, and voxelwise standard deviation measurements (Friedman and Glover, 2006; Glover et al., 2012). Preprocessing and functional data analysis were conducted within FSL 5.0.5 (Smith et al., 2004; http://www.fmrib.ox.ac.uk/fsl) and FEAT version 6.0. We also visually inspected the data for artifacts and blurring. Functional images were corrected for slice-timing and head motion using 6 rigid-body transformations with FSL MCFLIRT (Jenkinson et al., 2002). Using FSL motion outliers, motioncorrupted volumes where participants moved more than $2.5 \mathrm{~mm}$ in any direction within a run were modeled as confound variables, comprising less than one percent of volumes in total. Within each run, functional images were spatially normalized to the individual's FSPGR and co-registered to the MNI152 T1 template (Montreal Neurological Institute, Montreal, Canada) using a combination of affine and non-linear registrations (Greve and
Fischl, 2009; Jenkinson and Smith, 2001; Jenkinson et al., 2002). Images were spatially smoothed with a $5 \mathrm{~mm}$ Gaussian kernel, and a high-pass filter (cut off $=90.0 \mathrm{~s}$ ) was incorporated into the GLM to correct for scanner drift.

\subsubsection{Voxelwise analyses}

At the first level, the hemodynamic response of each event was modeled with a double $\gamma$ function for each participant. The design matrix of the first GLM included four independent events: negative images, neutral images, correct target trials within each condition, and errors (incorrect or omitted responses to targets), relative to an implicit baseline comprising the standards. There were ten regressors per run per person: three for the different trial types, one for errors, and six nuisance regressors representing motion. Furthermore, in a second GLM, we modeled correct target trials as a function of whether the most recent distractor image was negative (target after negative; TAneg) or neutral (target after neutral; TAneut), which included three independent events: target trials after negative images, target trials after neutral images, and target trials after neither image type. There were nine regressors per run per person: three for the different trial types, and six nuisance regressors representing motion. Within FSL, we made comparisons between trial types in order to identify differences in functional activation between levels of each variable. We modeled three comparisons within each run: the emotional valence of the distractor image-related activation from the negative $>$ neutral contrast. For target-related activation overall, targets were compared to baseline (standards). Estimation of the effect of emotional distraction on target processing was modeled as TAneg > TAneut.

At the second level, the three comparisons, negative $>$ neutral, targets $>$ baseline, and TAneg $>$ TAneut, were combined for each participant across all experimental runs, and also contrasting hard > easy and easy > hard. The resulting combinations of overall, hard > easy, and easy > hard for each of the effects of interest were analyzed with one sample t-tests at the group level using FMRIB Local Analysis of Mixed Effects (FLAME 1 \& 2) (Beckmann et al., 2003; Woolrich et al., 2004). In one sample t-tests with covariates, each behavioral measure, drift rate, nondecision time, and boundary separation, was coded as a demeaned continuous variable and run separately as a single regressor in the model. We used a cluster threshold of at $z>2.3$, GRF-corrected at $p<0.05$. All reported regions were identified and labeled using the Harvard-Oxford Atlas within FSL, and confirmed with other atlases (Desikan et al., 2006; Duvernoy, 1999). Coordinates reported are in MNI space, and results are overlaid on the MNI template brain. 


\section{Acknowledgements}

This research was supported by grants R01 MH098301 and R01 AG039684 from the National Institutes of Health. We are grateful to Drs. Allen Song, Michele Diaz, and Jie Zhuang for their assistance. The authors have no conflicts of interest to declare.

\section{References}

Alexopoulos, G.S., Hoptman, M.J., Kanellopoulos, D., Murphy, C.F., Lim, K.O. Gunning, F.M., 2012. Functional connectivity in the cognitive control network and the default mode network in late-life depression. J. Affect. Disord. 139, 5665.

Anderson, A.K., Christoff, K., Panitz, D., De Rosa, E., Gabrieli, J.D., 2003. Neura correlates of the automatic processing of threat facial signals. J. Neurosci. 23, 5627-5633.

Beckmann, C.F., Jenkinson, M., Smith, S.M., 2003. General multilevel linear modeling for group analysis in FMRI. Neuroimage 20, 1052-1063.

Bishop, S., Duncan, J., Brett, M., Lawrence, A.D., 2004. Prefrontal cortical function and anxiety: controlling attention to threat-related stimuli. Nat. Neurosci. 7 , $184-188$.

Bishop, S.J., 2008. Neural mechanisms underlying selective attention to threat. Ann N. Y. Acad. Sci. 1129, 141-152.

Blair, K.S., Smith, B.W., Mitchell, D.G., Morton, J., Vythilingam, M., Pessoa, L., Fridberg, D., Zametkin, A., Sturman, D., Nelson, E.E., Drevets, W.C., Pine, D.S., Martin, A., Blair, R.J., 2007. Modulation of emotion by cognition and cognition by emotion. Neuroimage 35, 430-440.

Blair, R.J., Morris, J.S., Frith, C.D., Perrett, D.I., Dolan, R.J., 1999. Dissociable neural responses to facial expressions of sadness and anger. Brain 122 (Pt 5), 883-893.

Chikazoe, J., Jimura, K., Asari, T., Yamashita, K., Morimoto, H., Hirose, S., Miyashita Y., Konishi, S., 2009. Functional dissociation in right inferior frontal cortex during performance of go/no-go task. Cereb. Cortex 19, 146-152.

Comte, M., Schon, D., Coull, J.T., Reynaud, E., Khalfa, S., Belzeaux, R., Ibrahim el, C., Guedj, E., Blin, O., Weinberger, D.R., Fakra, E., 2016. Dissociating bottom-up and top-down mechanisms in the cortico-limbic system during emotion processing. Cereb. Cortex 26, 144-155.

Cusack, R., Mitchell, D.J., Duncan, J., 2010. Discrete object representation, attention switching, and task difficulty in the parietal lobe. J. Cogn. Neurosci. 22, 32-47.

Desikan, R.S., Segonne, F., Fischl, B., Quinn, B.T., Dickerson, B.C., Blacker, D., Buckner, R.L., Dale, A.M., Maguire, R.P., Hyman, B.T., Albert, M.S., Killiany, R.J., 2006. An automated labeling system for subdividing the human cerebral cortex on MR scans into gyral based regions of interest. Neuroimage 31, 968-980.

Doallo, S., Holguin, S.R., Cadaveira, F., 2006. Attentional load affects automatic emotional processing: evidence from event-related potentials. NeuroReport 17 1797-1801.

Dolcos, F., Kragel, P., Wang, L., McCarthy, G., 2006. Role of the inferior frontal cortex in coping with distracting emotions. NeuroReport 17, 1591-1594.

Dolcos, F., Diaz-Granados, P., Wang, L., McCarthy, G., 2008. Opposing influences of emotional and non-emotional distracters upon sustained prefrontal cortex activity during a delayed-response working memory task. Neuropsychologia 46 , 326-335.

Dolcos, F., Iordan, A.D., Dolcos, S., 2011a. Neural correlates of emotion-cognition interactions: a review of evidence from brain imaging investigations. J. Cogn. Psychol. (Hove) 23, 669-694.

Dolcos, S., Sung, K., Denkova, E., Dixon, R.A., Dolcos, F., 2011b. Brain imaging investigation of the neural correlates of emotion regulation. J. Vis. Exp., e2430

Dosenbach, N.U., Visscher, K.M., Palmer, E.D., Miezin, F.M., Wenger, K.K., Kang, H.C., Burgund, E.D., Grimes, A.L., Schlaggar, B.L., Petersen, S.E., 2006. A core system for the implementation of task sets. Neuron 50, 799-812.

Dosenbach, N.U., Fair, D.A., Miezin, F.M., Cohen, A.L., Wenger, K.K., Dosenbach, R.A., Fox, M.D., Snyder, A.Z., Vincent, J.L., Raichle, M.E., Schlaggar, B.L., Petersen, S.E., 2007. Distinct brain networks for adaptive and stable task control in humans. Proc. Natl. Acad. Sci. U.S.A. 104, 11073-11078.

Duvernoy, H.M., 1999. The Human Brain: Surface, Blood Supply, and ThreeDimensional Sectional Anatomy. Springer-Verlag Wien, Vienna, Austria.

Eckert, M.A., Menon, V., Walczak, A., Ahlstrom, J., Denslow, S., Horwitz, A., Dubno, J. R., 2009. At the heart of the ventral attention system: the right anterior insula. Hum. Brain Mapp. 30, 2530-2541.

Folstein, M.F., Folstein, S.E., McHugh, P.R., 1975. "Mini-mental state”. A practical method for grading the cognitive state of patients for the clinician. J. Psychiatr. Res. 12, 189-198.

Friedman, L., Glover, G.H., 2006. Report on a multicenter fMRI quality assurance protocol. J. Magn. Reson. Imaging 23, 827-839.

Glover, G.H., Mueller, B.A., Turner, J.A., van Erp, T.G., Liu, T.T., Greve, D.N., Voyvodic, J.T., Rasmussen, J., Brown, G.G., Keator, D.B., Calhoun, V.D., Lee, H.J., Ford, J.M., Mathalon, D.H., Diaz, M., O'Leary, D.S., Gadde, S., Preda, A., Lim, K.O., Wible, C.G. Stern, H.S., Belger, A., McCarthy, G., Ozyurt, B., Potkin, S.G., 2012. Function biomedical informatics research network recommendations for prospective multicenter functional MRI studies. J. Magn. Reson. Imaging 36, 39-54.

Gomez, P., Ratcliff, R., Perea, M., 2007. A model of the go/no-go task. J. Exp. Psychol. Gen. 136, 389-413.

Greve, D.N., Fischl, B., 2009. Accurate and robust brain image alignment using boundary-based registration. Neuroimage $48,63-72$.
Gu, X., Han, S., 2007. Attention and reality constraints on the neural processes of empathy for pain. Neuroimage 36, 256-267.

Gur, R.C., Schroeder, L., Turner, T., McGrath, C., Chan, R.M., Turetsky, B.I., Alsop, D., Maldjian, J., Gur, R.E., 2002. Brain activation during facial emotion processing. Neuroimage 16, 651-662.

Hariri, A.R., Bookheimer, S.Y., Mazziotta, J.C., 2000. Modulating emotional responses: effects of a neocortical network on the limbic system. NeuroReport 11, 43-48

Hartikainen, K.M., Ogawa, K.H., Knight, R.T., 2000. Transient interference of right hemispheric function due to automatic emotional processing. Neuropsychologia 38, 1576-1580.

Hartikainen, K.M., Ogawa, K.H., Soltani, M., Knight, R.T., 2007. Emotionally arousing stimuli compete for attention with left hemispace. NeuroReport 18, 1929-1933.

Hartikainen, K.M., Ogawa, K.H., Knight, R.T., 2010. Trees over forest: unpleasant stimuli compete for attention with global features. NeuroReport 21, 344-348.

Hartikainen, K.M., Siiskonen, A.R., Ogawa, K.H., 2012. Threat interferes with response inhibition. NeuroReport 23, 447-450.

Herath, P., Klingberg, T., Young, J., Amunts, K., Roland, P., 2001. Neural correlates of dual task interference can be dissociated from those of divided attention: an fMRI study. Cereb. Cortex 11, 796-805.

Jenkinson, M., Smith, S., 2001. A global optimisation method for robust affine registration of brain images. Med. Image Anal. 5, 143-156.

Jenkinson, M., Bannister, P., Brady, M., Smith, S., 2002. Improved optimization for the robust and accurate linear registration and motion correction of brain images. Neuroimage 17, 825-841.

Kesler-West, M.L., Andersen, A.H., Smith, C.D., Avison, M.J., Davis, C.E., Kryscio, R.J., Blonder, L.X., 2001. Neural substrates of facial emotion processing using fMRI. Brain Res. Cogn. Brain Res. 11, 213-226.

Kober, H., Barrett, L.F., Joseph, J., Bliss-Moreau, E., Lindquist, K., Wager, T.D., 2008. Functional grouping and cortical-subcortical interactions in emotion: a metaanalysis of neuroimaging studies. Neuroimage 42, 998-1031.

Kramer, A.F., Madden, D.J., 2008. Attention. In: Craik, F.I.M., Salthouse, T.A. (Eds.), The Handbook of Aging and Cognition. Psychology Press, New York, pp. 189249

Kurth, S., Majerus, S., Bastin, C., Collette, F., Jaspar, M., Bahri, M.A., Salmon, E., 2016. Effects of aging on task- and stimulus-related cerebral attention networks. Neurobiol. Aging 44, 85-95.

Lange, K., Williams, L.M., Young, A.W., Bullmore, E.T., Brammer, M.J., Williams, S.C., Gray, J.A., Phillips, M.L., 2003. Task instructions modulate neural responses to fearful facial expressions. Biol. Psychiatry 53, 226-232.

Lesher, E.L., Berryhill, J.S., 1994. Validation of the Geriatric Depression Scale-Short Form among inpatients. J. Clin. Psychol. 50, 256-260.

Madden, D.J., Spaniol, J., Costello, M.C., Bucur, B., White, L.E., Cabeza, R., Davis, S.W., Dennis, N.A., Provenzale, J.M., Huettel, S.A., 2009. Cerebral white matter integrity mediates adult age differences in cognitive performance. J. Cogn. Neurosci. 21, 289-302.

Maki-Marttunen, V., Pickard, N., Solbakk, A.K., Ogawa, K.H., Knight, R.T. Hartikainen, K.M., 2014. Low attentional engagement makes attention network activity susceptible to emotional interference. NeuroReport 25, 1038-1043.

Maki-Marttunen, V., Kuusinen, V., Brause, M., Perakyla, J., Polvivaara, M., dos Santos Ribeiro, R., Ohman, J., Hartikainen, K.M., 2015. Enhanced attention capture by emotional stimuli in mild traumatic brain injury. J. Neurotrauma 32, 272-279.

Maki-Marttunen, V., Kuusinen, V., Perakyla, J., Ogawa, K.H., Brause, M., Brander, A. Hartikainen, K.M., 2017. Greater attention to task-relevant threat due to orbitofrontal lesion. J. Neurotrauma 34, 400-413.

Mather, M., Carstensen, L.L., 2003. Aging and attentional biases for emotional faces. Psychol. Sci. 14, 409-415.

Maylor, E.A., Lavie, N., 1998. The influence of perceptual load on age differences in selective attention. Psychol. Aging 13, 563-573.

McAvinue, L.P., Habekost, T., Johnson, K.A., Kyllingsbaek, S., Vangkilde, S., Bundesen, C., Robertson, I.H., 2012. Sustained attention, attentional selectivity, and attentional capacity across the lifespan. Atten Percept Psychophys. 74, 15701582 .

Monge, Z.A., Madden, D.J., 2016. Linking cognitive and visual perceptual decline in healthy aging: the information degradation hypothesis. Neurosci. Biobehav. Rev. 69, 166-173.

Morris, J.S., Ohman, A., Dolan, R.J., 1999. A subcortical pathway to the right amygdala mediating "unseen" fear. Proc. Natl. Acad. Sci. U.S.A. 96, 1680-1685.

Oldfield, R.C., 1971. The assessment and analysis of handedness: the Edinburgh inventory. Neuropsychologia 9, 97-113.

Pessoa, L., Kastner, S., Ungerleider, L.G., 2002a. Attentional control of the processing of neural and emotional stimuli. Brain Res. Cogn. Brain Res. 15, 31-45.

Pessoa, L., McKenna, M., Gutierrez, E., Ungerleider, L.G., 2002b. Neural processing of emotional faces requires attention. Proc. Natl. Acad. Sci. U.S.A. 99, 1145811463.

Petersen, S.E., Posner, M.I., 2012. The attention system of the human brain: 20 years after. Annu. Rev. Neurosci. 35, 73-89.

Phelps, E.A., 2006. Emotion and cognition: insights from studies of the human amygdala. Annu. Rev. Psychol. 57, 27-53.

Phillips, M.L., Young, A.W., Senior, C., Brammer, M., Andrew, C., Calder, A.J., Bullmore, E.T. Perrett, D.I., Rowland, D., Williams, S.C., Gray, J.A., David, A.S., 1997. A specific neural substrate for perceiving facial expressions of disgust. Nature 389, 495-498.

Pizzagalli, D.A., 2011. Frontocingulate dysfunction in depression: toward biomarkers of treatment response. Neuropsychopharmacology 36, 183-206. 
Ratcliff, R., 1978. A theory of memory retrieval. Psychol. Rev. 85, 59-108.

Ratcliff, R., Smith, P.L., Brown, S.D., McKoon, G., 2016. Diffusion decision model: current issues and history. Trends Cogn. Sci. 20, 260-281.

Ritchey, M., Dolcos, F., Cabeza, R., 2008. Role of amygdala connectivity in the persistence of emotional memories over time: an event-related FMRI investigation. Cereb. Cortex 18, 2494-2504.

Schmidt, L.J., Belopolsky, A.V., Theeuwes, J., 2015. Attentional capture by signals of threat. Cogn. Emot. 29, 687-694.

Shafer, A.T., Matveychuk, D., Penney, T., O'Hare, A.J., Stokes, J., Dolcos, F., 2012. Processing of emotional distraction is both automatic and modulated by attention: evidence from an event-related fMRI investigation. J. Cogn. Neurosci. 24, 1233-1252.

Smith, S.M., 2002. Fast robust automated brain extraction. Hum. Brain Mapp. 17, $143-155$.

Smith, S.M., Jenkinson, M., Woolrich, M.W., Beckmann, C.F., Behrens, T.E., JohansenBerg, H., Bannister, P.R., De Luca, M., Drobnjak, I., Flitney, D.E., Niazy, R.K., Saunders, J., Vickers, J., Zhang, Y., De Stefano, N., Brady, J.M., Matthews, P.M., 2004. Advances in functional and structural MR image analysis and implementation as FSL. Neuroimage 23 (Suppl 1), S208-S219.

Tomasi, D., Chang, L., Caparelli, E.C., Ernst, T., 2007. Different activation patterns for working memory load and visual attention load. Brain Res. 1132, 158-165.

Touroutoglou, A., Hollenbeck, M., Dickerson, B.C., Feldman Barrett, L., 2012. Dissociable large-scale networks anchored in the right anterior insula subserve affective experience and attention. Neuroimage 60, 1947-1958.

van Ravenzwaaij, D., Oberauer, K., 2009. How to use the diffusion model: Parameter recovery of three methods: EZ, fast-dm, and DMAT. J. Math. Psychol. 53, 463473.

Velanova, K., Jacoby, L.L., Wheeler, M.E., McAvoy, M.P., Petersen, S.E., Buckner, R.L., 2003. Functional-anatomic correlates of sustained and transient processing components engaged during controlled retrieval. J. Neurosci. 23, 8460-8470.

Vincent, J.L., Kahn, I., Snyder, A.Z., Raichle, M.E., Buckner, R.L., 2008. Evidence for a frontoparietal control system revealed by intrinsic functional connectivity. J. Neurophysiol. 100, 3328-3342.

Voss, A., Nagler, M., Lerche, V., 2013. Diffusion models in experimental psychology: a practical introduction. Exp. Psychol. 60, 385-402.
Voyvodic, J.T., 1999. Real-time fMRI paradigm control, physiology, and behavior combined with near real-time statistical analysis. Neuroimage 10, 91-106.

Wagenmakers, E.J., van der Maas, H.L., Grasman, R.P., 2007. An EZ-diffusion mode for response time and accuracy. Psychon. Bull. Rev. 14, 3-22.

Wagenmakers, E.J. van der Maas, H.L., Dolan, C.V., Grasman, R.P., 2008. EZ does it! Extensions of the EZ-diffusion model. Psychon. Bull. Rev. 15, 1229-1235.

Walter, H., Adenzato, M., Ciaramidaro, A., Enrici, I., Pia, L., Bara, B.G., 2004 Understanding intentions in social interaction: the role of the anterior paracingulate cortex. J. Cogn. Neurosci. 16, 1854-1863.

Wang, L., McCarthy, G., Song, A.W., Labar, K.S., 2005. Amygdala activation to sad pictures during high-field (4 tesla) functional magnetic resonance imaging. Emotion 5, 12-22.

Wang, L., LaBar, K.S., McCarthy, G., 2006. Mood alters amygdala activation to sad distractors during an attentional task. Biol. Psychiatry 60, 1139-1146.

Wang, L., Huettel, S., De Bellis, M.D., 2008a. Neural substrates for processing taskirrelevant sad images in adolescents. Dev. Sci. 11, 23-32.

Wang, L., Krishnan, K.R., Steffens, D.C., Potter, G.G., Dolcos, F., McCarthy, G., 2008b. Depressive state- and disease-related alterations in neural responses to affective and executive challenges in geriatric depression. Am. J. Psychiatry $165,863-871$.

Wang, L., LaBar, K.S., Smoski, M., Rosenthal, M.Z., Dolcos, F., Lynch, T.R., Krishnan, R.R. McCarthy, G., 2008c. Prefrontal mechanisms for executive control over emotional distraction are altered in major depression. Psychiatry Res. 163, 143-155.

Weissman, D.H., Gopalakrishnan, A., Hazlett, C.J., Woldorff, M.G., 2005. Dorsa anterior cingulate cortex resolves conflict from distracting stimuli by boosting attention toward relevant events. Cereb. Cortex 15, 229-237.

Woolrich, M.W., Behrens, T.E., Beckmann, C.F., Jenkinson, M., Smith, S.M., 2004 Multilevel linear modelling for FMRI group analysis using Bayesian inference. Neuroimage 21, 1732-1747.

Yang, Y., Bender, A.R., Raz, N., 2015. Age related differences in reaction time components and diffusion properties of normal-appearing white matter in healthy adults. Neuropsychologia 66, 246-258.

Yarkoni, T, Gray, J.R., Chrastil, E.R., Barch, D.M. Green, L., Braver, T.S., 2005. Sustained neural activity associated with cognitive control during temporally extended decision making. Brain Res. Cogn. Brain Res. 23, 71-84. 